\title{
Arterial Spin-Labeled Perfusion Imaging Reflects Vascular Density in Nonfunctioning Pituitary Macroadenomas
}

\author{
N. Sakai, S. Koizumi, S. Yamashita, Y. Takehara, H. Sakahara, S. Baba, Y. Oki, H. Hiramatsu, and H. Namba
}

\begin{abstract}
BACKGROUND AND PURPOSE: Angiogenesis is very important in clinical features of pituitary adenomas. We investigated the relationship between the blood flow of nonfunctioning pituitary macroadenomas measured by arterial spin-labeled perfusion imaging and the microvessel attenuation of the tissue.

MATERIALS AND METHODS: Conventional MR imaging with contrast-enhanced TIWI and arterial spin-labeled perfusion imaging were performed before surgery in 11 consecutive patients with nonfunctioning pituitary macroadenomas. ROIs were drawn on the tumors, and the degrees of enhancement were calculated by dividing the signal intensity on the contrast-enhanced TIWI by that on the nonenhanced TIWI. As an index of tumor perfusion, a quantitative analysis was performed by using normalized tumor blood flow values calculated by dividing the mean value of the tumor region of interest by the mean region of interest values in the 2 cerebellar hemispheres. The relative microvessel attenuation was determined as the total microvessel wall area divided by the entire tissue area on CD-31-stained specimens. The degree of enhancement and the normalized tumor blood flow values were compared with relative microvessel attenuation. Additionally, intra- and postoperative tumor hemorrhages were visually graded.

RESULTS: The degree of enhancement was not correlated with relative microvessel attenuation. Statistically significant correlations were observed between normalized tumor blood flow values and relative microvessel attenuation $(P<.05)$. At surgery, 3 cases were visually determined to be hypervascular tumors, and 1 of these cases had symptomatic postoperative hemorrhage. A statistically significant difference in normalized tumor blood flow values was observed visually between the intraoperative hypovascular and hypervascular groups $(P<.05)$.
\end{abstract}

CONCLUSIONS: Arterial spin-labeled perfusion imaging reflects the vascular density of nonfunctioning pituitary macroadenomas, which may be useful in the preoperative prediction of intra- and postoperative tumor hemorrhage.

ABBREVIATIONS: ASL-PI = arterial spin-labeled perfusion imaging; $\mathrm{nTBF}=$ normalized tumor blood flow; $\mathrm{TBF}=$ tumor blood flow; \% Vessel = relative microvessel attenuation

$\mathbf{P}$

ituitary adenomas are common intracranial neoplasms, with up to $16.7 \%$ of the population harboring an incidental tumor. ${ }^{1}$ Although most pituitary adenomas grow slowly, some may begin to grow rapidly and become invasive. ${ }^{2}$ Solid tumors are dependent on the process of angiogenesis for growth. ${ }^{3}$ The measurement of microvessel attenuation has been shown to be a useful quantitative method for assessing angiogenesis. ${ }^{2}$ The assessment of the differences in vascular density in pituitary adenomas may be helpful for predict-

Received November 1, 2012; accepted after revision January 26, 2013.

From the Departments of Neurosurgery (N.S., S.K., H.H., H.N.), Radiology (S.Y., Y.T., H.S.), Diagnostic Pathology (S.B.), and Endocrinology and Metabolism (Y.O.), Hamamatsu University School of Medicine, Hamamatsu, Japan.

Please address correspondence to Naoto Sakai, MD, PhD, Department of Neurosurgery, Hamamatsu University School of Medicine, 1-20-1, Handayama, Higashi-ku, Hamamatsu, Japan 431-3192; e-mail: nsakaineurosurg@gmail.com

http://dx.doi.org/10.3174/ajnr.A3564 ing subsequent tumor aggressiveness ${ }^{4-6}$ and vascular complications associated with transsphenoidal surgery. ${ }^{7,8}$

Arterial spin-labeled perfusion imaging is a noninvasive MR perfusion technique that measures CBF by using arterial water as a freely diffusing tracer without the requirement of exogenous contrast material. ${ }^{9}$ In clinical applications, ASL-PI has been proved reliable and reproducible for the assessment of CBF in various pathologic states, ${ }^{1}$ including cerebrovascular diseases,${ }^{10,11}$ neurodegenerative diseases, ${ }^{12}$ and brain tumors. ${ }^{13,14}$ ASL-PI may predict histopathologic vascular density ${ }^{15}$ and may reflect angiogenesis in tumors. The purpose of our study was to determine the relationship between the blood flow of nonfunctioning pituitary macroadenomas measured by using ASL-PI and histopathologic findings of microvessel attenuation. Furthermore, we examined the utility of ASL-PI in predicting intra- and postoperative tumor hemorrhage. 
Summary of patient characteristics, histopathologic findings, tumor volume and enhancement, microvessel density, and intra- and postoperative hemorrhages in 11 patients with nonfunctioning pituitary macroadenomas

\begin{tabular}{|c|c|c|c|c|c|c|c|c|c|}
\hline $\begin{array}{c}\text { Case/Age } \\
(\mathrm{yr}) / \text { Sex }\end{array}$ & $\begin{array}{c}\text { Initial/ } \\
\text { Recurrence }\end{array}$ & Histopathology & $\begin{array}{c}\text { Tumor } \\
\text { Volume } \\
(\mathrm{cm})\end{array}$ & $\begin{array}{c}\text { Degree of } \\
\text { Enhancement } \\
\text { of Tumor }\end{array}$ & $\begin{array}{l}\text { ASL-PI } \\
\text { nTBF }\end{array}$ & $\begin{array}{c}\text { ASL-PI TBF } \\
(\mathrm{mL} / \mathrm{min} / 100 \mathrm{~g})\end{array}$ & $\begin{array}{c}\text { CD-31 } \\
\text { \%Vessel }\end{array}$ & $\begin{array}{l}\text { Intraoperative } \\
\text { Vascularity } \\
\text { Findings }\end{array}$ & $\begin{array}{c}\text { Postoperative } \\
\text { Hemorrhage }\end{array}$ \\
\hline $1 / 72 / M$ & Initial & SGA & 11.4 & 1.48 & 1.94 & 94.4 & 24.0 & $(+)$ & $(+)^{a}$ \\
\hline $2 / 52 / M$ & Recurrence & NCA & 8.7 & 1.50 & 0.47 & 29.5 & 1.3 & $(-)$ & $(-)$ \\
\hline $3 / 63 / M$ & Initial & SGA & 4.7 & 1.36 & 0.96 & 22.7 & 0.7 & $(-)$ & $(-)$ \\
\hline $4 / 48 / F$ & Recurrence & SGA & 2.2 & 1.44 & 0.75 & 51.0 & 1.7 & $(-)$ & $(-)$ \\
\hline $5 / 54 / M$ & Initial & SGA & 6.0 & 1.71 & 1.88 & 74.2 & 17.0 & $(+)$ & $(+)$ \\
\hline $6 / 69 / M$ & Recurrence & $\mathrm{NCA}$ & 7.7 & 1.81 & 0.72 & 26.6 & 2.6 & $(-)$ & $(-)$ \\
\hline $7 / 60 / F$ & Initial & NCA & 2.8 & 1.27 & 0.51 & 38.0 & 1.1 & $(-)$ & $(-)$ \\
\hline $8 / 34 / F$ & Initial & SGA & 4.9 & 1.88 & 0.73 & 59.5 & 1.2 & $(-)$ & $(-)$ \\
\hline $9 / 68 / M$ & Recurrence & $\mathrm{NCA}$ & 3.4 & 1.82 & 0.76 & 26.6 & 2.6 & $(-)$ & $(-)$ \\
\hline $10 / 60 / F$ & Initial & NCA & 9.9 & 1.68 & 2.45 & 102.6 & 19.0 & $(+)$ & $(+)$ \\
\hline $11 / 67 / M$ & Recurrence & SGA & 4.7 & 1.96 & 1.03 & 46.2 & 6.4 & $(-)$ & $(-)$ \\
\hline
\end{tabular}

Note:-SGA indicates gonadotroph adenoma; NCA, null cell adenoma; ASL-PI nTBF, tumor blood flow normalized to the cerebellum measured with ASL-PI; ASL-PI TBF, absolute tumor blood flow (milliliters per minute per $100 \mathrm{~g}$ ) measured with ASL-PI; (-), hypovascular; +, hypervascular.

a Symptomatic hemorrhage.

\section{MATERIALS AND METHODS \\ Patients}

Between June 2011 and March 2012, eleven consecutive patients (7 men and 4 women) with pituitary macroadenomas were admitted to the University Hospital of Hamamatsu University School of Medicine for surgery. All patients were evaluated by 1 endocrinologist (Y.O.) and were diagnosed as having clinically nonfunctioning pituitary adenomas. They underwent ASL-PI with conventional MR imaging and MR angiography. Written informed consent was obtained from all patients. The mean age of the patients was 58 years (range, 34-72 years). Six cases were of initial macroadenomas, and 5 showed recurrences. The chief concerns were visual disturbance in 9 cases and headache and amenorrhea in 1 case each. All patients underwent transsphenoidal surgery performed by a single neurosurgeon (N.S.). All surgical procedures were recorded by using a digital video recorder (DATA Gen Pro; Seventh Dimension Design, Hyogo, Japan) and were reviewed for this study. Intraoperative tumor hemorrhage was rated as hypovascular or hypervascular at surgery by 2 neurosurgeons (N.S. and S.K.) in consensus. "Hypovascular tumors" were defined as tumors with a normal amount of arterial bleeding that rapidly decreased during the process of resection. "Hypervascular tumors" were defined as tumors with a higher-than-normal amount of arterial bleeding that continued throughout the operation. Postoperative hemorrhage from the residual tumor or pituitary gland was estimated by using MR imaging studies performed within 2 days after the operation. The hematoma volume was visually assessed by 2 experienced radiologists (S.Y. and Y.T.) in consensus on sagittal T1WI with fat suppression.

\section{Conventional MR Imaging, MRA, ASL-PI, and ASL \\ Data Processing}

Conventional MR imaging studies for pituitary adenomas and MRA were performed on a 1.5T scanner (Signa HDx TwinSpeed; GE Healthcare, Milwaukee, Wisconsin) with an 8-channel brain phased array coil or a 3T scanner (Discovery 750; GE Healthcare) with a 32-channel head-neck-spine array coil.

The sequences of conventional MR imaging included coronal and sagittal fat-suppressed T1WI, fat-suppressed T2WI, and contrast-enhanced fat-suppressed T1WI. The imaging parameters for

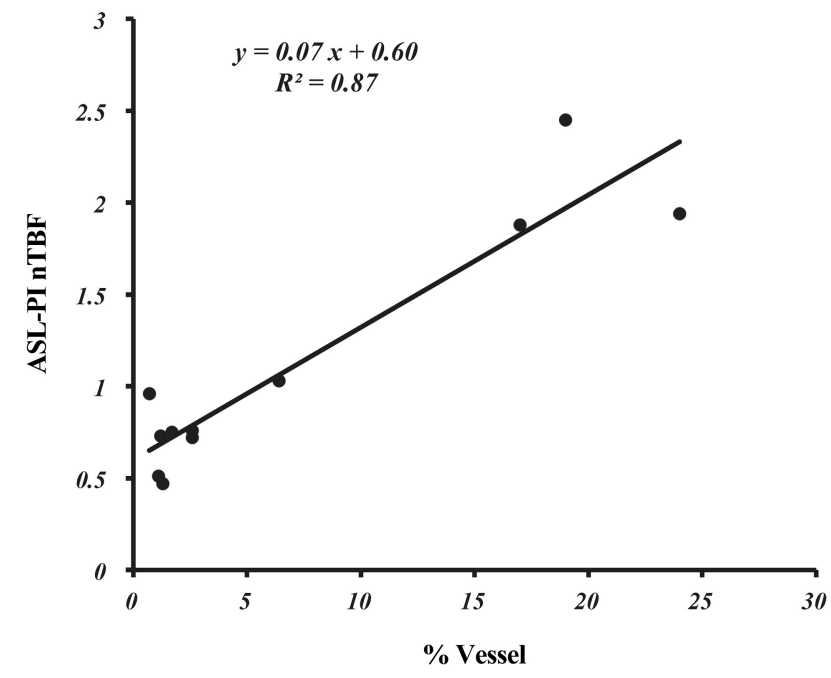

FIG 1. Scatterplot of nTBF determined by arterial spin-labeled perfusion imaging and \%Vessel determined by histopathologic examination with regression lines in 11 patients with nonfunctioning pituitary macroadenomas. The correlation between normalized tumor blood flow and \%Vessel is statistically significant $(P<.05)$ (Spearman rankorder correlation).

the 1.5T scanner were as follows: TR/ TE/NEX, $600 \mathrm{~ms} / 9.4 \mathrm{~ms} / 2$; receiver bandwidth, $22.7 \mathrm{kHz}$; section thickness, $3 \mathrm{~mm}$; spacing, $0.3 \mathrm{~mm}$; number of sections, 9; FOV, $15 \mathrm{~cm}$; matrix, $192 \times 160$ for the fat-suppressed T1WI; and TR/TE/NEX, $3000 \mathrm{~ms} / 101.3 \mathrm{~ms} / 2$; receiver bandwidth, $15.6 \mathrm{kHz}$; section thickness, $3 \mathrm{~mm}$; spacing, $0.3 \mathrm{~mm}$; number of sections, 9; FOV, $15 \mathrm{~cm}$; and matrix, $192 \times$ 160 for the fat-suppressed T2WI. The imaging parameters of the 3T scanner were as follows: TR/TE/NEX, $600 \mathrm{~ms} / 9.2 \mathrm{~ms} / 3$; receiver bandwidth, $41.7 \mathrm{kHz}$; section thickness, $3 \mathrm{~mm}$; spacing, 0.3 mm; number of sections, 9; FOV, $16 \mathrm{~cm}$; and matrix, $320 \times 192$ for the fat-suppressed T1WI; and TR/TE/NEX, $2000 \mathrm{~ms} / 82.7$ $\mathrm{ms} / 2$; receiver bandwidth, $41.7 \mathrm{kHz}$; section thickness, $3 \mathrm{~mm}$; spacing, $0.3 \mathrm{~mm}$; number of sections, 9; FOV, $16 \mathrm{~cm}$; matrix, $320 \times 224$ for the fat-suppressed T2WI. Fat-suppressed contrastenhanced T1WI was performed after intravenous administration of gadopentetate dimeglumine (Magnevist; Bayer Schering Pharma, Berlin, Germany) or gadodiamide hydrate (Omniscan; 

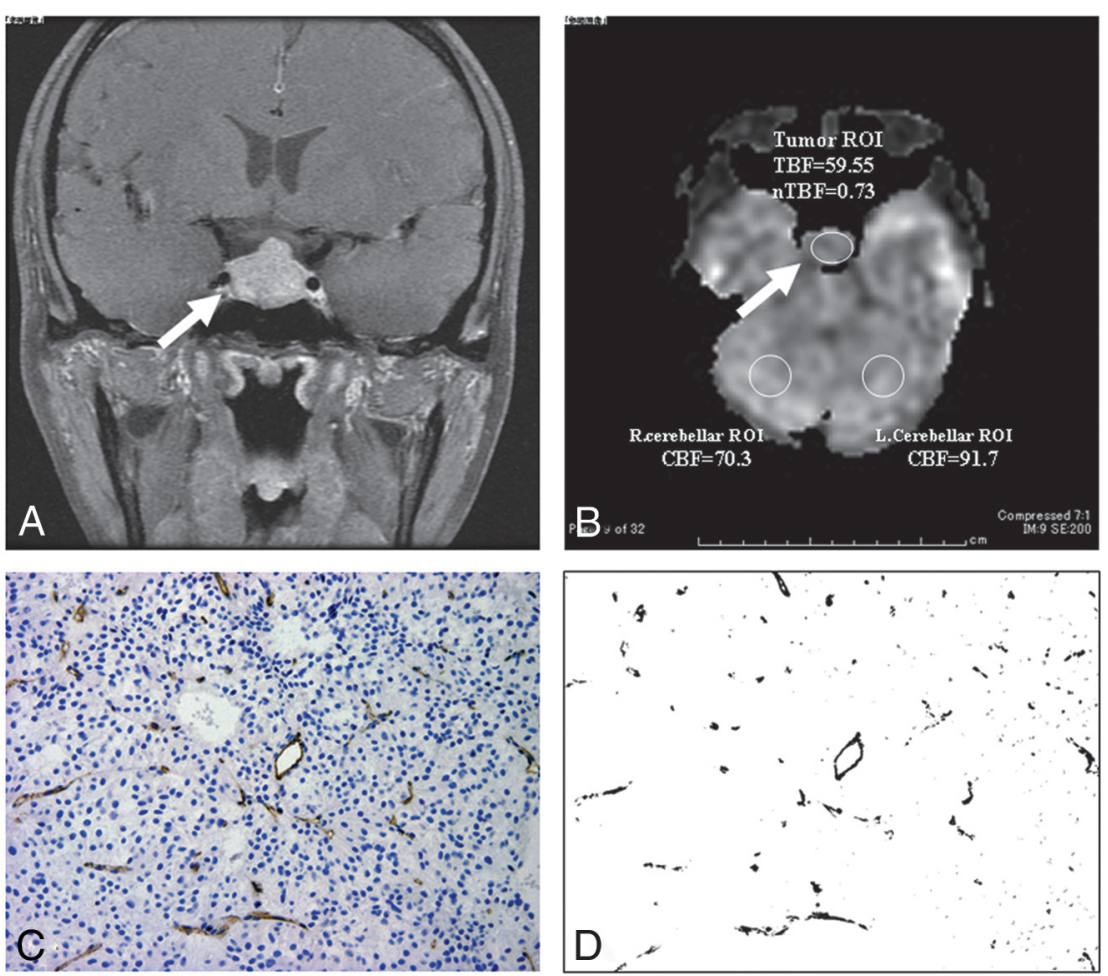

FIG 2. Nonfunctioning pituitary macroadenoma with hypovascularity in a 34-year-old woman (case 8). A, Coronal postcontrast fat-suppressed TIWI (TR/TE/NEX $=600 \mathrm{~ms} / 9.4 \mathrm{~ms} / 2$ ) by using a 1.5T scanner shows a strongly enhanced pituitary tumor (arrow) (degree of enhancement $=$ 1.88). $B$, Arterial spin-labeled perfusion imaging (TR/TE/NEX $=4594 \mathrm{~ms} / 10.6 \mathrm{~ms} / 3$; postlabel delay $=1525 \mathrm{~ms}$; section thickness $=3.8 \mathrm{~mm}$ ) by using a $3 \mathrm{~T}$ scanner shows a relatively low signal intensity at the pituitary tumor (arrow) (TBF $=59.5 \mathrm{~mL} / \mathrm{min} / 100 \mathrm{~g}, \mathrm{nTBF}=0.73) . \mathrm{nTBF}=$ $\mathrm{TBF} /$ (Right Cerebellar CBF + Left Cerebellar CBF)/2. The CD-31-immunostained histopathologic specimen $(C)$ and the black on the vessel wall area $(D)$ indicate low microvascular density $(\%$ Vessel $=1.2 \%)$

Daiichi-Sankyo Pharma, Tokyo, Japan) at $0.1 \mathrm{mmol} / \mathrm{kg}$ body weight. For contrast-enhanced images, the degree of enhancement was calculated by dividing the mean signal intensity of the region of interest drawn on the tumor on contrast-enhanced fatsuppressed T1WI by the signal intensity in the region of interest drawn on the macroadenoma on the nonenhanced fat-suppressed T1WI. Tumor volumes (cubic centimeters) were calculated by multiplying the vertical and anteroposterior and right-to-left maximum dimensions on postcontrast T1WI and subsequently dividing this value by 2 .

We performed 3D time-of-flight MRA on all patients before surgery to confirm that obstructive lesions were not present at the cervical common and internal carotid arteries and intracranial arteries. We used the following parameters: TR/TE/NEX, $30 \mathrm{~ms} / 2.7 \mathrm{~ms} / 0.75$; flip angle, 20; receiver bandwidth, $31.2 \mathrm{kHz}$; section thickness, 1.2 $\mathrm{mm}$; overlap, $0.6 \mathrm{~mm}$; number of sections, 176; FOV, $20 \mathrm{~cm}$; and matrix, $288 \times 192$ on the $1.5 \mathrm{~T}$ scanner; and TR/TE/NEX, $18 \mathrm{~ms} / 2.6$ $\mathrm{ms} / 1$; flip angle, 18; receiver bandwidth, $41.7 \mathrm{kHz}$; section thickness, $1 \mathrm{~mm}$; overlap, $0.5 \mathrm{~mm}$; number of sections, 208; FOV, 20 $\mathrm{cm}$; and matrix, $320 \times 192$ on the $3 \mathrm{~T}$ scanner.

All ASL-PI examinations were performed on the 3T scanner. The imaging parameters were as follows: TR/TE/NEX, $4594 \mathrm{~ms} /$ $10.6 \mathrm{~ms} / 3$; receiver bandwidth, $62.5 \mathrm{kHz}$; postlabel delay, 1525 ms; section thickness, $3.8 \mathrm{~mm}$; number of sections, 32; FOV, 24 $\mathrm{cm}$; and matrix, 512. These parameters have been fixed in the routine clinical setting at our university hospital for stroke and brain tumors. We performed a pilot study to compare CBF measured by ASL-PI and brain perfusion SPECT in 7 cases of internal carotid artery stenosis. In these cases, a significant linear correlation was obtained between the CBF measured by ASL-PI and the CBF measured by SPECT (coefficient of correlation $=0.5$; H.H., unpublished data, December 2012). Therefore, we used the same parameters for ASL-PI in this study. The region of interest was located at the maximal axial cross-section of the pituitary adenomas by referring to the findings on contrast-enhanced fat-suppressed T1WI. To evaluate normalized tumor blood flow, we calculated tumorto-healthy tissue perfusion ratios by dividing the mean value of the tumor region of interest by the mean value of the ROIs in the bilateral cerebellar hemispheres (approximately $2.5 \mathrm{~cm}^{2}$ each) by following the method of Järnum et al (Figs $2 B$ and $3 B){ }^{14}$

\section{Histopathologic Evaluations}

Hematoxylin-eosin-stained sections and immunohistochemical stains against adrenocorticotrophic hormone, prolactin, growth hormone, luteinizing hormone, follicle-stimulating hormone, and thyroid-stimulating hormone were examined by 1 pathologist (S.B.). The samples were classified as null cell adenoma or silent gonadotroph adenoma. We immunostained the tissue sections with CD-31, the most sensitive and specific marker for endothelial cells, to evaluate the vascularity of the nonfunctioning pituitary adenomas. Sections for CD-31 staining were pretreated with Tris-EDTA buffer, $\mathrm{pH} 9.0$, at $95^{\circ} \mathrm{C}$ for 40 minutes for antigen activation. The monoclonal mouse antihuman CD-31 antibody, clone JC70A (M0823; Dako, Glostrup, Denmark), was applied at a dilution of 1:20 for 30 minutes at room temperature, and the ChemMate EnVision Detection Kit Peroxidase/DAB, Rabbit/Mouse Autostainer (Dako) was used for staining. The CD-31-positive endothelial cell layers indicated the vessel walls. The relative microvessel attenuation (\%Vessel) was calculated as the total area of the vessel walls divided by the entire tissue area by following the method of Noguchi et al. ${ }^{15} \mathrm{~A}$ microscopic field of the most intense vascularization in each specimen under a $\times 20$ objective field (area $=0.33 \mathrm{~mm}^{2}$ ) was photographed as a JPEG file $(1600 \times 1200$ pixels, 16.7 million colors, 8 bit) by using a microscopic digital color camera. The JPEG files were converted to TIFF files by using Photoshop (Adobe Systems, San Jose, California). The images were analyzed by using ImageJ 1.45s (National Institutes of Health, Bethesda, Maryland) to measure \%Vessel by counting the pixel number of the total vessel walls and that of the entire tissue sample. 

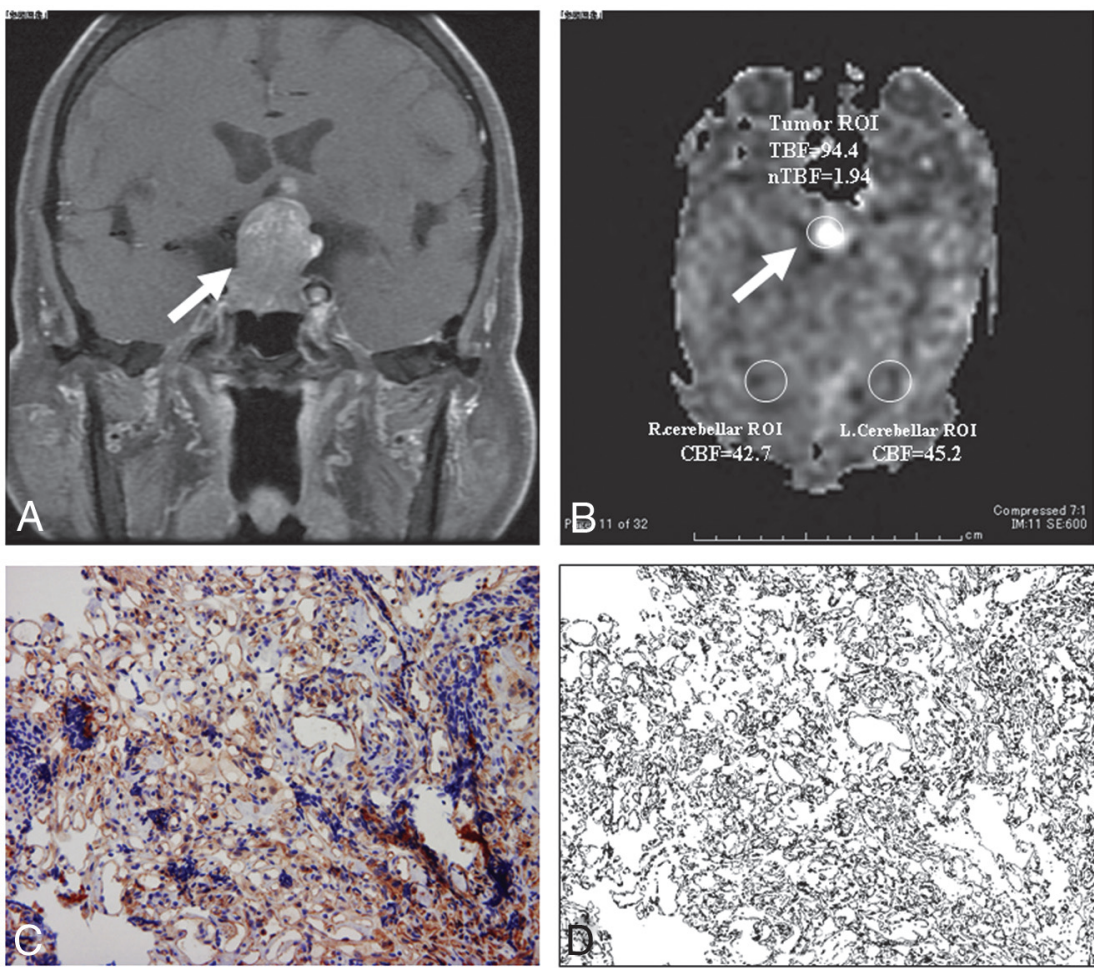

FIG 3. Nonfunctioning pituitary macroadenoma with hypervascularity in a 72-year-old man (case 1) in whom a postoperative symptomatic hemorrhage with visual deterioration required an emergent reoperation to resolve the visual disturbance. $A$, Coronal postcontrast fat-suppressed TIWI (TR/TE/NEX $=600 \mathrm{~ms} / 9.4 \mathrm{~ms} / 2$ ) by using a $1.5 \mathrm{~T}$ scanner shows a strongly enhanced pituitary tumor (arrow) (degree of enhancement $=1.48$ ). $B$, Arterial spin-labeled perfusion imaging (TR/TE/NEX $=4594 \mathrm{~ms} / 10.6 \mathrm{~ms} / 3$; postlabel delay $=1525 \mathrm{~ms}$; section thickness $=3.8$ $\mathrm{mm}$ ) by using a 3T scanner shows a relatively high signal intensity at the pituitary tumor (arrow) $(\mathrm{TBF}=94.4 \mathrm{~mL} / \mathrm{min} / 100 \mathrm{~g}, \mathrm{nTBF}=1.94) . \mathrm{nTBF}=\mathrm{TBF} /$ (Right Cerebellar CBF + Left Cerebellar CBF)/2. The CD-31-immunostained histopathologic specimen (C) and the black on the vessel wall area $(D)$ indicate high microvascular density (\%Vessel $=24 \%$ ).

\section{Statistical Analysis}

An Excel Statistical Program File ystat2008.xls (programmed by Shinya Yamazaki, DDS, PhD; Igaku-Tosho-Shuppan, Tokyo, Japan) was used for analysis.

The correlation between nTBF and tumor volume was evaluated by using the Spearman rank-order correlation. The differences in nTBF between intraoperative hypovascular and hypervascular tumors were compared by using the Mann-Whitney $U$ test. The correlations between the degree of enhancement and $\%$ Vessel and between nTBF and \%Vessel were evaluated by using the Spearman rank-order correlation. Probability values $<.05$ were considered significant.

\section{RESULTS}

Patient characteristics, histopathologic findings, tumor volumes, the degree of enhancement of tumors, ASL-PI nTBF, ASL-PI TBF (milliliter per minute per $100 \mathrm{~g}$ ), \%Vessel of CD-31, and findings of intraoperative tumor vascularity and postoperative hemorrhage are summarized in the Table. The tumor volumes ranged from 2.2 to $11.4 \mathrm{~cm}^{3}$ (mean, $6.0 \pm 3.0 \mathrm{~cm}^{3}$ ). The degree of enhancement of the tumors ranged from 1.27 to 1.96 (mean, $1.63 \pm$ 0.23). The ASL-PI nTBF ranged from 0.47 to 2.45 (mean, $1.11 \pm$ 0.66), and the ASL-PI TBF ranged from 22.7 to 102.6 (mean, $51.9 \pm 27.9) \mathrm{mL} / \mathrm{min} / 100 \mathrm{~g}$. The $\%$ Vessel of CD-31 ranged from
$0.7 \%$ to $24 \%$ (mean, $7.1 \pm 8.6 \%$ ). Five cases were classified as null cell adenomas; and 6, as silent gonadotroph adenomas. A statistically significant correlation was not observed between ASL-PI nTBF and tumor volume. The degree of enhancement was statistically correlated with neither ASL-PI nor \%Vessel.

Statistically significant differences in ASL-PI nTBF were observed between hypovascular tumors and hypervascular tumors $(P<.05)$. At surgery, 8 cases were determined to be hypovascular tumors, and 3 cases were determined to be hypervascular. The postoperative MR imaging did not reveal any hemorrhages in the hypovascular tumors. On the other hand, all of the hypervascular tumors exhibited postoperative hemorrhage that filled the space after tumor removal. An emergent reoperation the day after the surgery was required for one of these patients because of visual acuity deterioration due to compression by the hematoma. The patient's visual acuity fully recovered after the surgery.

The correlation between ASL-PI nTBF and \%Vessel in the 11 cases is shown in Fig 1. Statistically significant positive correlations were found between ASL-PI nTBF and \%Vessel ( $\mathrm{x}, \%$ Vessel; $\mathrm{y}$, ASL-PI nTBF; $\left.\mathrm{y}=0.07 \mathrm{x}+0.60, R^{2}=0.87, P \leq .05\right)$.

A representative case of a hypovascular tumor (case 8) and a hypervascular tumor (case 1) are shown in Figs 2 and 3, respectively. A distinctly high signal was observed in the hypervascular pituitary tumor with high microvessel attenuation on ASL-PI (Fig 3; $\mathrm{TBF}=94.4 \mathrm{~mL} / \mathrm{min} / 100 \mathrm{~g}, \mathrm{nTBF}=1.94$, and CD-31\%Vessel $=$ $24.0 \%)$. However, a nonspecific signal equivalent to the background cerebellar hemispheres was observed in the hypovascular pituitary tumor with low microvessel attenuation (Fig 2; $\mathrm{TBF}=59.5 \mathrm{~mL} / \mathrm{min} /$ $100 \mathrm{~g}, \mathrm{nTBF}=0.73$, and $\mathrm{CD}-31 \%$ Vessel $=1.2 \%)$. The degree of enhancement was 1.48 in the hypervascular tumor (case 1) and 1.88 in the hypovascular tumor (case 8).

\section{DISCUSSION}

The ASL-PI technique has been developed since its inception in the early 1990s and is now used routinely in daily clinical practice for patients with stroke. ${ }^{9}$ This technique is noninvasive, does not require injection of an exogenous contrast agent, and was recently shown to evaluate the perfusion in brain tumors. ${ }^{13-15}$ Although the absolute blood flow values could be theoretically determined by ASL-PI, ${ }^{9}$ the quantitative CBF determined by ASL-PI can be affected by unstable factors in individual cases such as the arterial transit time of the labeled blood from the labeling plane to the brain tissue within the imaging plane. ${ }^{15}$ Lower perfusion with a longer transit time in elderly subjects results in underestimation of the CBF because the distal end of the labeled bolus does not reach the capillary bed. ${ }^{13}$ Therefore, the TBF has been normalized 
to healthy tissue such as the contralateral gray matter, ${ }^{13,15}$ white matter, ${ }^{13}$ or the cerebellum ${ }^{14}$ in ASL-PI studies. In this study, we chose the cerebellum as the internal reference because cerebellar perfusion usually is unaffected by pathology in the pituitary gland and is easier to measure in the same imaging plane with pituitary adenomas. ${ }^{14}$

In our study, 3 of 11 cases were categorized as hypervascular tumors on the basis of intraoperative findings, and the nTBF of the hypervascular tumors was significantly higher than that of the hypovascular tumors $(P<.05)$. Furthermore, all of the hypervascular tumors with a high nTBF showed postoperative hemorrhage. These findings suggest that ASL-PI is useful for predicting intra- and postoperative tumor hemorrhage. Preoperative evaluation of ASL-PI provides useful information for avoiding or preparing for the vascular complications of transsphenoidal surgery, which can lead to mortality and serious morbidity. 7,8

We found a strong positive correlation between nTBF and \%Vessel in the 11 patients with nonfunctioning pituitary macroadenomas, suggesting that ASL-PI reflects the vascular density of nonfunctioning pituitary macroadenomas. To date, the source of the blood supply to pituitary tumors has not been fully elucidated. ${ }^{2}$ A portion of the blood supply to tumors is presumably derived from the branches of the internal carotid artery, most commonly the capsular and inferior hypophyseal arteries. ${ }^{16}$ The sequential enhancement pattern of pituitary adenomas detected during a dynamic contrast- enhanced study suggests that pituitary adenomas have a direct arterial blood supply. ${ }^{17}$ We speculate that ASL-PI measures the arterial blood flow of the pituitary adenoma that is derived mainly from the branches of the internal carotid artery and, to some extent, from the external carotid artery. However, further studies are necessary to characterize the blood supply of pituitary tumors fully.

Although we observed a trend toward a positive correlation between nTBF and tumor volumes in this study, this correlation did not reach statistical significance. Further studies with a larger sample size are necessary to reveal the chronologic changes of the arterial blood supply to the tumor reflected by nTBF in the process of tumor enlargement.

All of the 11 cases in this study showed strong contrast enhancement. If the contrast agent was confined to the intravascular space and caused a signal enhancement proportional to its concentration, the degree of enhancement could have reflected the vascular density of the tumors. However, nonspecific gadolinium chelates rapidly equilibrate between the intravascular and extracellular matrix of tumors. ${ }^{18}$ This phenomenon may primarily explain why the degree of enhancement did not linearly correlate with the vascular density in this study.

The lack of a quantitative analysis of intraoperative tumor hemorrhage was a limitation of our study. The decision regarding tumor vascularization depended on the surgeon's subjective measurement. Intraoperative angiography by using indocyanine green fluorescence with a quantitative analysis may be helpful for evaluating tumor vascularization. The low spatial resolutions and limited section coverage of the present ASL-PI were major technical limitations. The measurement of CBF in the normal pituitary gland and microadenomas is difficult by the present methods. The difference in the feeding arteries between the internal carotid artery and the external carotid artery may have affected the timing of the bolus arrival, possibly resulting in bias. ASL-PI with selective labeling of the external carotid artery ${ }^{19}$ may resolve this bias. Finally, possible disagreements existed between the measurement region of nTBF and \%Vessel in the histopathologic specimen.

\section{CONCLUSIONS}

Our study results revealed a positive correlation between nTBF and $\%$ Vessel in nonfunctioning pituitary macroadenomas, suggesting that ASL-PI reflects the vascular density of nonfunctioning pituitary macroadenomas. ASL-PI may be useful in the preoperative prediction of intra- or postoperative tumor hemorrhage.

\section{REFERENCES}

1. Ezzat S, Asa SL, Couldwell WT, et al. The prevalence of pituitary adenomas. Cancer 2004;101:613-19

2. Turner HE, Harris AL, Melmed S, et al. Angiogenesis in endocrine tumors. Endocr Rev 2003;24:600-32

3. Folkman J. Seminars in medicine of the Beth Israel Hospital, Boston: clinical applications of research on angiogenesis. $N$ Engl J Med 1971;285:1182-86

4. Jugenburg M, Kovacs K, Stefaneanu L, et al. Vasculature in nontumorous hypophyses, pituitary adenomas, and carcinomas: a quantitative morphologic study. Endocr Pathol 1995;6:115-24

5. Turner HE, Nagy ZN, Gatter KC, et al. Angiogenesis in pituitary adenomas and the normal pituitary gland. J Clin Endocrinol Metab 2000;85:1159-62

6. de la Torre NG, Turner HE, Wass JA. Angiogenesis in prolactinoma: regulation and relationship with tumor behaviour. Pituitary 2005;8:17-23

7. Laws ER Jr. Vascular complications of transsphenoidal surgery. Pituitary 1999;2:163-70

8. Berker M, Aghayev K, Saatci I, et al. Overview of vascular complications of pituitary surgery with special emphasis on unexpected abnormality. Pituitary 2010;13:160-67

9. Deibler AR, Pollock JM, Kraft RA, et al. Arterial spin-labeling in routine clinical practice. Part 1 . Technique and artifacts. AJNR Am J Neuroradiology 2008;29:1228-34

10. Wang DJ, Aloger JR, Quiao JX, et al. The value of arterial spin-labeled perfusion imaging in acute ischemic stroke: comparison with dynamic susceptibility contrast-enhanced MRI. Stroke 2012;43:1018-24

11. Bokkers ROH, Hermandez DA, Merino HJ, et al. Whole-brain arterial spin labeling perfusion in patients with acute stroke. Stroke 2012;43:1290-94

12. Alsop DC, Detre JA, Grossman M. Assessment of cerebral blood flow in Alzheimer's disease by spin-labeled magnetic resonance imaging. Ann Neurol 2000;47:93-100

13. Warmuth C, Günther M, Zimmer C. Quantification of blood flow in brain tumors: comparison of arterial spin labeling and dynamic susceptibility-weighted contrast-enhanced MR imaging. Radiology 2003;228:523-32

14. Järnum H, Steffensen EG, Knutsson L, et al. Perfusion MRI of brain tumours: a comparative study of pseudo-continuous arterial spin labeling and dynamic susceptibility contrast imaging. Neuroradiology 2010;52:307-17

15. Noguchi T, Yoshiura T, Hiwarashi A, et al. Perfusion imaging of brain tumors using arterial spin-labeling: correlation with histopathologic vascular density. AJNR Am J Neuroradiol 2008;29:688-93

16. Powell DF, Baker HL, Laws ER. The primary angiographic findings in pituitary adenomas. Radiology 1974;110:589-95

17. Yuh WT, Fisher DJ, Nguyen HD, et al. Sequential MR enhancement pattern in normal pituitary gland and in pituitary adenoma. AJNR Am J Neuroradiol 1994;15:101-08

18. Roberts TPL, Roberts HC. Macromolecular contrast agents. In: Dawson P, Cosgrove DO, Grainger RG, eds. Textbook of Contrast Media. San Francisco: Isis Medical Media Ltd; 1999:355-71

19. Sasao A, Hirai T, Nishimura S, et al. Assessment of vascular supply of hypervascular extra-axial brain tumors with $3 \mathrm{~T}$ MR regional perfusion imaging. AJNR Am J Neuroradiol 2010;31:554-58 